\title{
Implementation of the care programme approach in the community
}

\author{
Chris Gilleard
}

\begin{abstract}
The comprehensive implementation of the care programme approach is described within a large Mental Health Unit serving two London boroughs. Methods used to introduce an incremental improvement in the care programme approach (CPA) proctice and documentation are outlined together with an account of the development of a set of quality standards and a CPA annual audit system.
\end{abstract}

The care programme approach (CPA) was formally outlined in the Department of Health circular HC (90) 23 (Department of Health, 1990). The main requirements of the approach have been described by Kingdon (1994). The essential features are that:

(a) it applies to all patients accepted for treatment and care by specialist mental health services

(b) it identifies a plan or programme of care tailored to the individual social and clinical circumstances of each patient

(c) it identifies a key worker responsible for coordinating the interventions and care plans in the care programme

(d) it identifies a review process to ensure that the care plans are effective and up to date.

Plans to implement the approach were discussed within our Mental Health Unit by a working group which had been developing guidelines for multidisciplinary community mental health teams. It was agreed that we should aim to implement the approach systematically across all teams and services, irrespective of their level of multidisciplinary working and team cohesiveness.

\section{The Unit wide approach to local implementation}

Local guidelines for implementing the CPA were first drawn up in March 1991, requiring that a care plan/programme be drawn up and recorded for all patients irrespective of which service they were in and who was involved in their care. The care programme should clearly state the name of the key worker, other professionals involved in the care of the patient, the components of the care plan and a review date. A copy was to be kept in the notes and a copy sent to the general practitioner (GP).

Initially, there were major difficulties in completing the necessary documentation for most of the catchment area mental health teams. Team information systems were inadequate and in some cases lacking altogether, communication between team members was limited and the management of patients' notes often complex and disjointed. A series of seminars about the care programme approach were held during September 1991 to discuss the Unit's operational guidelines and the difficulties teams were experiencing with them. The main areas of concern focused upon completing the documentation, the roles and responsibilities of key workers and the nature of the care plan.

In December 1991 the first audit exercise took place. The notes of all patients discharged during October 1991 into community settings, and all patients referred to four of the twelve catchment area mental health teams in November 1991 were examined. Of the discharged patients, $46 \%$ had no recorded care programme with no obvious reason why this should be so. Of the newly referred community patients, $35 \%$ had no recorded care programme with no reason for this.

We identified as minimal standards the existence of a documented care programme, a named key worker and a planned review date. Approximately $25 \%$ of the care programmes 
met these minimal standards-including those of the patients discharged to the community and those referred in the community.

Following this report a revised set of local guidelines was drawn up and circulated in December 1992. These sought to clarify the means of registering patients accepted for treatment and care, the expectations of being a key worker, in what form the care programme should be documented and the conceptual separation of specific clinical care plans from the overall coordinated care programme. Arrangements for a further series of seminars were organised around the new guidelines.

The next audit sweep incorporated a retrospective review of the notes of all patients discharged during the month of October 1992 , and $a$ one in ten sample of the then current (October 1993) community caseloads of the 11 catchment area adult mental health teams (two adult CMHTs had merged between audits), the two catchment area elderly mental health teams and the two rehabilitation teams.

Of the 134 notes of patients discharged in the target month 104 (78\%) were traced and monitored by a team of three senior clinicians. Of the 2730 patients recorded on the 15 teams' caseloads, 290 casenotes were randomly identified. Monitoring of this intended community sample was carried out on 214 casenotes (74\%). Thirty-one per cent of the community patients had no documented care programme in their notes, with no evident reason for this being so; this was the case for $30 \%$ of the discharged patients' notes. Only $25 \%$ of the care programmes in the in-patients' notes met the minimal standards (a documented care programme, a named key worker and a planned review date). However, $63 \%$ of the community patients' casenotes met these standards - a marked improvement from the $25 \%$ success rate in the previous year.

The results of the second audit were presented in the form of a report which was widely circulated within the Unit. Individual meetings were held with each community mental health team during Spring 1994 to discuss their performance as indicated by the monitoring exercise and any problems arising either from the accuracy and interpretation of the figures themselves or from the set of recommendations contained within the second audit report. These meetings took place with most of the professions present in the teams (psychiatrists, nurses, psychologists, therapy staff, and attached social workers).
Implications for conducting a third audit were discussed, including the standards for documenting the CPA and the need to balance Unit wide auditing of these standards with team specific plans to conduct their own clinical audit of CPA.

\section{The current audit}

The above resumé of how CPA has been developed and implemented on a Unit wide basis across all the local mental health service teams in Merton and Wandsworth provides the context for reporting the results of the most recent audit conducted in November and December 1994.

The sampling frame was enlarged. The casenotes of every other patient on the teams' caseloads were to be monitored by a team of seven clinician/managers. Five sets of casenotes were independently rated by each member of the audit team against the monitoring standards derived from the recommendations in the second audit report. Any disagreements were discussed and ambiguities about the standards were clarified. Over a two month period each rater rated the caseloads of at least two teams. Altogether 1387 sets of casenotes were monitored, although the monitoring forms of four of these were lost. Just over 3000 cases were identified via the information department as being on the teams' current caseloads, but in several cases these were out of date, or referred to patients seen but not 'accepted' for treatment and/or care. Overall just under $50 \%$ of the estimated current caseloads of the teams were sampled, although the figures were below $45 \%$ in three teams and above $50 \%$ in two. Of the 1384 casenotes examined, $340(25 \%)$ had a reason why there was no active care programme (e.g. seen but not accepted; unrecorded discharge; referred on to another service; no longer in the community).

Nine key standards were monitored. The standards and the extent to which they were met are outlined in Table 1 and the figures compared with the one in ten sample from the previous year's monitoring of community team caseloads.

We examined the monitoring sheets to identify the clinicians who were involved in delivering care to the 787 community patients with recorded care programmes. Fifty-two per cent were recorded as receiving care from one mental health professional only, $30 \%$ by two mental health professionals, $11 \%$ by three 
Table 1. Standards of CPA documentation

\begin{tabular}{lll}
\hline Standard & $\begin{array}{l}\text { \% met (1994) } \\
(\mathbf{n = 1 0 4 4 )}\end{array}$ & $\begin{array}{l}\% \text { met (1993) } \\
(\mathbf{n = 2 1 4 )}\end{array}$ \\
\hline A documented care programme in the notes & 75 & 63 \\
Of those with a care programme, had they: & 97 & 88 \\
A named key worker & 73 & 63 \\
A planned review date & 80 & 85 \\
A standard form recording the programme & 96 & 85 \\
A clear set of care plans or objectives & 83 & 67 \\
Named persons responsible for each plan & 78 & 50 \\
A programme dated and signed by the kenworker & 27 & 0 \\
Evidence the programme had been sent to the GP & 73 & 63 \\
Minimal standards met & &
\end{tabular}

and $7 \%$ by four or more mental health professionals. There was a systematic relationship between the number of mental health professionals involved and the extent to which the care programme was recorded as arising from a multidisciplinary discussion/ meeting. While a third [33\%] of the care programmes with only one professional involved had a record of a multidisciplinary discussion, nearly $80 \%$ of the care programmes involving four or more professionals were recorded as having been drawn up in a multidisciplinary setting.

\section{Discussion}

The care programme approach provides a framework for good practice in the treatment and care of people suffering from mental illness (Kingdon, 1994). Accepting a patient for treatment and care requires a clear plan, often a number of interlinked plans, which need to be coordinated and which need to be regularly reviewed. The more people involved in a patient's care the more important it is to know who is responsible for which aspects of care or treatment.

The present audit has demonstrated the practicability of implementing CPA comprehensively across all areas of adult mental health, and for all patients accepted by the specialist mental health services. The process has involved a Unit wide acceptance of the principles of CPA, a clear direction and plan for implementation and regular review and feedback with all the services concerned. Although the quality of care offered to patients is not directly determined by the adequacy of its documentation, clearly documented care/ treatment plans must increase the likelihood that patients will receive a reasonable standard of care.
The benefits of implementing CPA comprehensively mean that common standards of documenting care apply irrespective of the service used and the clinicians involved, that multidisciplinary discussion of a patient's care occurs more often than not in all service areas, that individual clinical responsibilities for various aspects of care become clearer, and that patients and their GPs have an accessible record of what is being done and by whom. While it may be true that much of this was happening before CPA was introduced, in many areas it was not. Matters are no worse and in all probability a lot better since the policy was introduced. Unlike the case with many other aspects of health policy, nobody will make the headlines for receiving inadequate care and treatment as a result of the CPA.

\section{Acknowledgement}

I would like to thank Paul Brewer who has contributed significantly to the planning and implementation of CPA audit within our Unit for the last four years.

\section{References}

DEPARTMENT of HEALTH (1990) The Care Programme Approach for People with a Mental Miness Referred to the Specialist Psychiatric Services. Joint Health/Social Services circular HC (90) 23/LASSL (90) 11.

- (1993) Health of the Nation Key Area Handbook: Mental Illness. London: HMSO.

KINGDON, D. (1994) Care programme approach: recent government policy and legislation. Psychiatric Bulletin. 18. 68-70.

Chris Gilleard, Director of Psychology, Pathfinder Mental Health Services NHS Trust, Springfield University Hospital, Tooting, London SW17 7DJ 